\title{
Cost-Effective Access Based Android System of Mobile Application for Blind Person
}

\author{
Hilal A. Fadhil, Feras N. Hasoon, Suresh Manic K
}

\begin{abstract}
l:---nowadays, savvy purchasing in current shopping center stores that supply exclusive items is an fantastic check for humans with visual disabilities. A evidence-of-idea wearable framework imagined to help outwardly weakened clients with finding racked objects. the broadcast content cloth, as an example, scanner tag indicates up all around the vicinity. along the ones lines, dazzle individuals need a few help to peruse this standardized identification at the same time as goes out on the town to preserve. This paper suggests a digital camera-based totally definitely object facts peruser to assist daze people to peruse statistics about the gadgets thru the scanner tag. The virtual digital camera is going about as a primary imaginative and prescient in spotting the standardized identity of the items then the scanner tag will be organized inner to get the object statistics via manner of utilising ECLIPSETM programming by the usage of unfastened programming equipment PhpMyAdmin eventually distinguishes the object name, cost and brand. The identified item statistics is articulated via the content material todiscourse innovation (TTS). The TTS are utilized to change over the content into discourse regions with the aid of utilising voice yield. Its plan does no longer require any device instrumentation of the store and turns on low set up order and protection costs.
\end{abstract}

Catchphrases: scanner tag, dazzle patron, shopping for, content to-discourse

\section{CREATION}

As of late, there are various styles of matters especially sustenances, can be recognized through odor or touch, for instance, merchandise of the soil 2]. This shape of nourishment is straightforward for the visually impaired humans to differentiate, but it'll finishes up extra enthusiastically if the gadgets that bought at the buying complicated have an equivalent shape, for example, packing containers of chilies, canned pasta, oat boxes, bundles of sugar and others. a few different innovation primarily based Android application is normally implemented in outstanding utility to overcome the gadget impediments [3].the ones are a essential trouble of people with visible disabilities particularly the individuals who are visually impaired to be able to separate amongst holders when the cubicles which are held will enjoy identical but have an exchange substance [4]. notwithstanding the truth that they may be guided with everyday visually impaired route help, for instance, stick or getting ready hound, here and there it's far futile because the items often trade its region and surely do now not separate among exceptional forms of the gadgets. aside from that, it

Revised Manuscript Received on August 19, 2019.

Hilal A. Fadhil,Computer Eng. Dep. Al-Farabi University College, Baghdad-Iraq.

Feras N Hasoon., Dep. of Electrical and Computer Eng., National University of Science and Tech., Muscat, Oman.

,Suresh Manic K, Dep. of Electrical and Computer Eng., National University of Science and Tech., Muscat, Oman. may offer a hard hassle to the visually impaired humans on the off threat that they not get the perfect object, however introduced the gadgets which might be poisonous substance and dangerous, as an instance, the distinctive between paste live with the cope with stick. commonly, if an character goes for getting, daze people need to maintain up a store consultant to have a free minute in an effort to help them to apprehend the objects which they have been maintaining. this may load the 2 gatherings because the visually impaired people want to hang tight for a few minutes and want to painting the correct devices, whilst the store representative need to go through wonderful occasions to get for the item. From this condition, the visually impaired humans need to format their purchasing time throughout the time it is much less humans to come back, as an instance, working time. besides, this furthermore will set off diminishing the visually impaired humans freedom in day by day bodily activities. In view of the lately dispensed papers there are two sorts of searching out food: little scale and huge scale [5-6]. In little scale buying, the patron purchases only a couple of factors that may be conveyed by way of hand or in a hand bin. massive scale purchasing includes shopping for more objects and will regularly require the utilization of a buying basket. In a few different examination, we utilized the ergonomics-for one system [7] to speak with ten humans with visible obstacles about their trying to find food encounters $[8,9]$. This paper shows a financially savvy approach so as to plan a eager framework that assist the visually impaired client.

\section{LITERATURE EVALUATE}

Later paintings has tended to the difficulty of perusing standardized tags from pics gotten through manner of a virtual camera flexible smartphone[3-4]. A large portion of this art work has rearranged the problem of locating and fragmenting the scanner tag inside the image via using accepting sure imperatives preserve. $\operatorname{In}[1]$, the standardized identification is notion to be flat in the picture and saw close to enough for the lengthy width of the scanner tag to subtend round $66 \%$ of the photograph width; al-but the creators name interest to that the method can be effectively reached out to the example of vague path, it's miles vague how well the calculation could feature with standardized identifications observed from greater far off away. other paintings [2] accept that the standardized tag may be

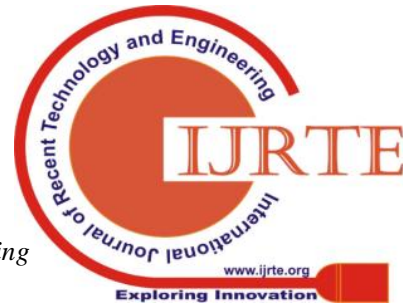


recognized from the morphological form of pairs regions however the method can also moreover flop on pics which is probably noisier than the crisp.[3] Assumes that a scanner tag can be identified through seeking out a dark bar using a winding technique, which outputs in a winding outward from the focus of the photo, yet it is not glaring that an individual dark bar may be competently all round settled a long way off. We expand on our ongoing paintings [4] concentrating on perusing a standardized identity (accepting it has just been fragmented) utilizing a Bayesian deformable format calculation that consolidates an in advance version of the geometric u .s .a .of the scanner tag design with a hazard model that assesses evidence inside the image for edges. Such anapproach is a giant system of interpreting boisterous scanner tag photographs that comprise fake (or lacking) edges. A associated methodology [5], moreover relying on debilitate succesful format, can efficaciously disentangle standardized tags from amazingly foggy and loud pics.

\section{DEVICE DESIGN}

Android framework has been clarified inside the in recent times dispensed paper in [6-8]. The proposed flowchart of our plan has been displayed in discern 1 . It demonstrates the calculation technique, the initial step is starts with the analyzing of the standardized identity of the object; at that issue, the framework desires to apprehend the Barcode this is the precept step towards the transfer united states of america of the HTML database. in addition, within the event that any information is decided, at that thing it desires to studies for the presence database refreshed item weight. Likewise, at the off risk that the recognized Bar code data is coordinating with item database weight, at that element it wishes to allow patron to maintain with voice yield and could report the message to patron about the purchasing, sold form of objects and entire invoice of objects. At very last, at the off threat that any bungle has been took place in looking at the weight, at that point it will produce the blunder message

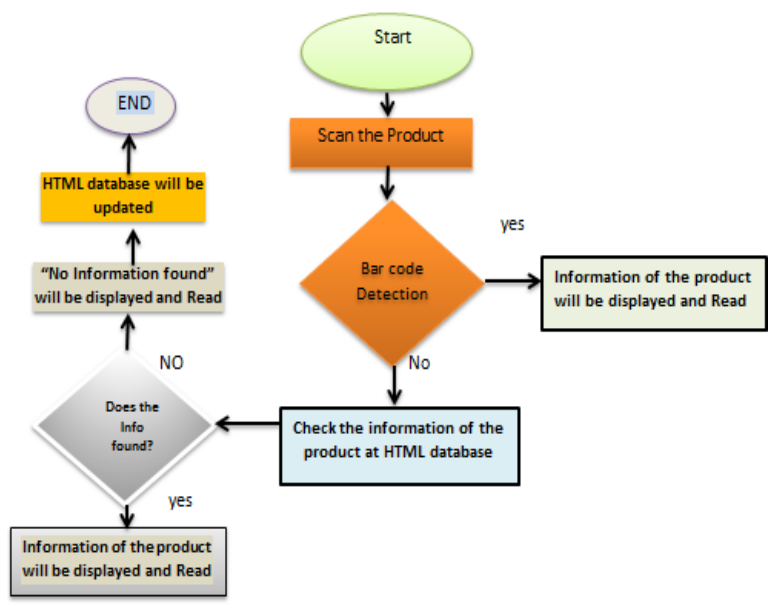

Fig.1: Proposed system flowchart

\section{BARCODEDETECTION}

Barcode can be represented as a line with various spacing and width, fashioned with the aid of dimensional styles of white and black shapes which representation of the statistics. This sample contains the information of the product. exceptall of the records given, the barcode additionally begin with a special individual and also ends with a unique individual. these codes can assist the reader to come across the barcode whether it is being scanned forward or backward. The barcode shape can be seen in determine 2 [6].

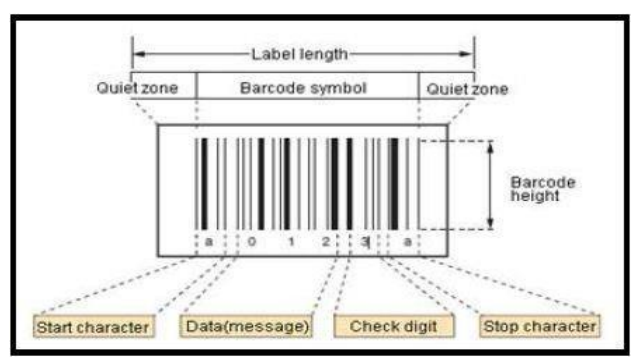

Figure 2 Bar code Structure

The detection function starts even as the amount of barcode is being scan, then looking for the data of the product in the database which has been already saved. This order will start finding the particular code wide variety by using populate in listing View sorts. however, this sort of detection high-quality can artwork for the product that used EAN13, EAN8, UPCA and UPCE sorts of barcode. discern 3 indicates the placing for the barcode software detection via using EclipseTM software software.

scanner.setConfig(Symbol.EAN13, Config.ENABLE,1); scanner.setConfig(Symbol.EAN8, Config.ENABLE,1); scanner.setConfig(Symbol.UPCA, Config.ENABLE,1); scanner.setConfig(Symbol.UPCE, Config.ENABLE,1);

\section{Fig.3: barcode program setting}

\section{TEXT-TO-SPEECHTECHNOLOGY}

These days, content to-discourse innovation is as of now notably applied for android stage as it's considered as a smooth approach to look for a few thing without making an funding time and vitality for perusing especially for those humans with seen weaknesses. in this challenge, Google software program has been implemented makes use of as a technique for coordinating with cell cellphone for showing the statistics of the item within the event that any precise offer. This technique has been related and modified into this venture internal to get the item data with the aid of the usage of ECLIPSETM programming via unfastened programming device PhpMyAdminTM. figure four demonstrates an example software program for the sound the use of content fabric to-discourse (TTS) device.

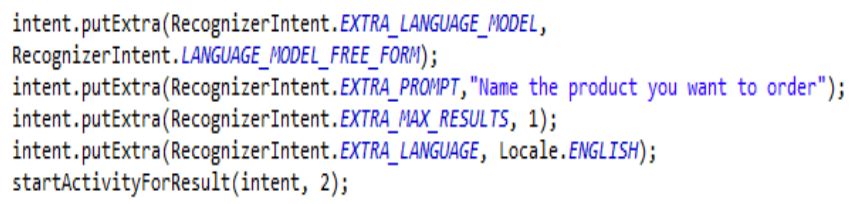

Fig.4: Audio TTS program
Published By:

Blue Eyes Intelligence Engineering \& Sciences Publication 


\section{EXPERIMENTALRESULTS}

The primary standardized identity vicinity and disentangling writing computer applications become created and actualized on a pc and in a while ported to the Lenovo S650 digicam phone. In our shape, the application have become accomplished to preserve running as fast as practicable at the cell smartphone with a view to system with the standardized identification images. to accomplish this, the framework's UI publications the client thru two degrees for each item, first locating and after that perusing a scanner tag. To assure exquisite air dissemination, the framework is hooked up a twine casing linked to the outside of the rucksack. The relaxation of the components - the standardized tag scanner's base station, the USB center point, and all associating links - are set within the knapsack's pocket. due to the fact the framework gives discourse based commands to the consumer, the framework has a touch earphone as seemed in Fig.5. The purchaser holds the virtual camera spherical over the outdoor of the object, grade by gradechecking the possible areas of the standardized identity; the framework is straightforwardly regarded till it discovers suitable sufficient evidence for a scanner tag in its concern of view. on the point whilst a standardized identification is prominent, the framework sound will declare the records of the item that has been output consisting of the call of item, kind, and value in USD \$. Likewise, a reproduction of information which has recognized by internet cam might be interpreted as content material cloth sheet at the pc as seemed in figure 6 . At prolonged final, a similar programming can be protected over cellphone bendy and via using related the device into the database of the precise shopping middle shops; the device can end up aware of the standardized identification information successfully as seemed in decide 7 .

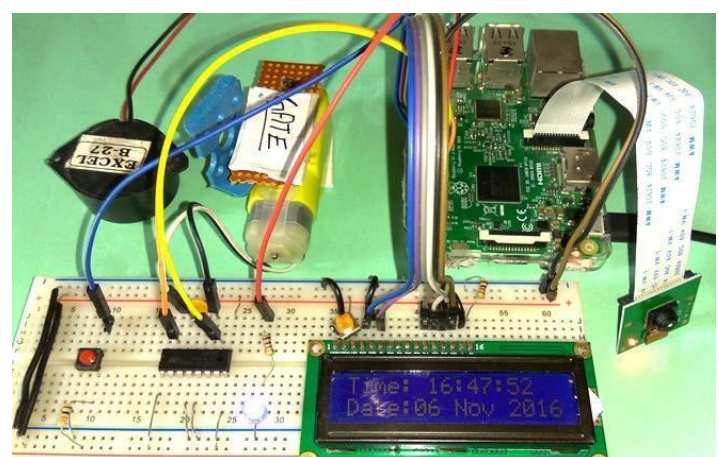

Figure 5 Proposed Hardware with barcode voice detection

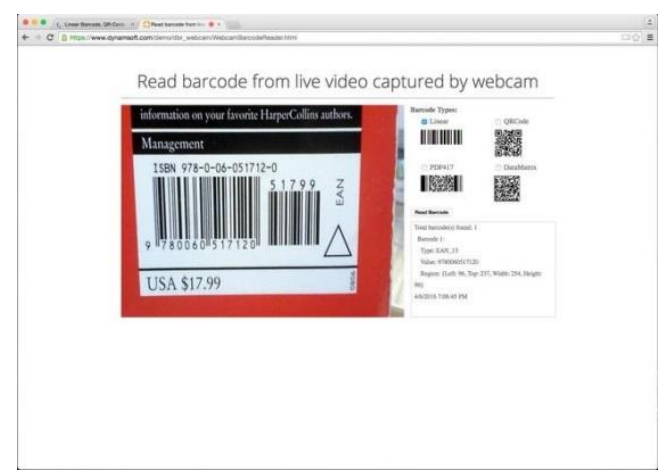

Figure 6 Barcode text info

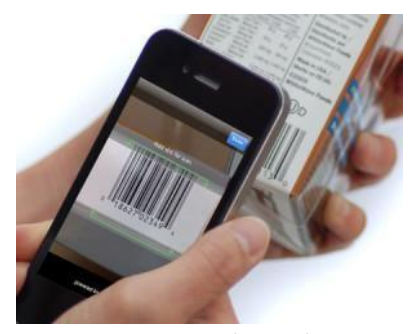

Figure 7 Barcode detection via Phone mobile

\section{CONCLUSION}

On this undertaking, a sensible methodology depends and actualized on every different framework depending on cellular cellular telephone that may causes the visually impaired clients to discover and peruse scanner tags thru making use of the digicam of the cellular smartphone. The product writing pc packages is incorporated with the framework gadget by means of webcam; finished it with the utility that has been actualized as a product utility. concurrently, the proposed framework can claim the price of the object with the resource of voice and content material fabric moreover. This framework is viewed as an optionally available eager approach to triumph over the issue that want to be looked by way of the visually impaired patron at doing their day by day buying motion particularly whilst is going by myself.

\section{REFERENCES}

1. Ni LM, Liu Y, Lau YC, Patil AP. LANDMARC: indoor vicinity detecting the use of dynamic RFID. WirelNetw 2004; 10(6): 701-10.

2. Gaunet $F$, Briffault $X$. Investigating the utilitarian determinations of a constrained wayfinding verbal guide for visually impaired human beings taking walks: basic and prepared city zones. Murmur Comput engage 2005; 20(3) 267-314

3. Kulyukin V, Nicholson J, Ross D, Marston J, GaunetF. The clueless leaders and their even greater clueless fans: towards synergistic on line path statistics the executives by way of humans with seen disabilities. Papers from the AAAI Spring Symposium 2008.

4. Leeb SB, Hovorka GB, Lupton EC, et al. Assistive correspondence frameworks for crippled people utilising unmistakable lighting. In: 15th international generation and people with Disabilities conference. Northridge, CA 2000.

5. Harter A, Hopper A, Steggles P, Ward A, Webster P. The existence systems of a contextaware software. WirelsNetw 2002; 8(2/3): 187-ninety seven.

6. Priyantha NB, Chakraborty A, Balakrishnan H. The cricket place emotionally supportive community. In: MobiCom '00: proceedings ofthe6th annualglobal accumulating on cell processing and structures administration. new york, massive apple, u.s.: ACM 2000; pp. 32-forty three.

7. LaMarca A, Chawathe Y, Consolvo S, et al. Spot lab: device situating utilising radio reference factors in nature. In: proceedings of the 0.33 international convention on Pervasive Computing. talk Notes in computer era. Springer-Verlag 2005; pp. 116-33.

8. Welbourne E, Lester J, LaMarca A, Borriello G. flexible setting induction using minimal try sensors. In: Strang $\mathrm{T}$, Linnhoff-Popien C, Eds. LoCA. vol. 3479 of Lecture Notes in laptop technology. Springer 2005; pp. 254-263. 